\title{
Generation Method of Multipiecewise Linear Chaotic Systems Based on the Heteroclinic Shil'nikov Theorem and Switching Control
}

\author{
Chunyan Han, ${ }^{1,2}$ Fang Yuan, ${ }^{3}$ and Xiaoyuan Wang ${ }^{3}$ \\ ${ }^{1}$ School of Automation, Guangdong University of Technology, Guangzhou 510006, China \\ ${ }^{2}$ Department of Photoelectric Engineering, Binzhou University, Binzhou 256603, China \\ ${ }^{3}$ School of Electronics and Information, Hangzhou Dianzi University, Hangzhou 310018, China \\ Correspondence should be addressed to Chunyan Han; cyh660@163.com
}

Received 31 March 2015; Accepted 3 June 2015

Academic Editor: René Yamapi

Copyright ( 2015 Chunyan Han et al. This is an open access article distributed under the Creative Commons Attribution License, which permits unrestricted use, distribution, and reproduction in any medium, provided the original work is properly cited.

\begin{abstract}
Based on the heteroclinic Shil'nikov theorem and switching control, a kind of multipiecewise linear chaotic system is constructed in this paper. Firstly, two fundamental linear systems are constructed via linearization of a chaotic system at its two equilibrium points. Secondly, a two-piecewise linear chaotic system which satisfies the Shil'nikov theorem is generated by constructing heteroclinic loop between equilibrium points of the two fundamental systems by switching control. Finally, another multipiecewise linear chaotic system that also satisfies the Shil'nikov theorem is obtained via alternate translation of the two fundamental linear systems and heteroclinic loop construction of adjacent equilibria for the multipiecewise linear system. Some basic dynamical characteristics, including divergence, Lyapunov exponents, and bifurcation diagrams of the constructed systems, are analyzed. Meanwhile, computer simulation and circuit design are used for the proposed chaotic systems, and they are demonstrated to be effective for the method of chaos anticontrol.
\end{abstract}

\section{Introduction}

In 1994, Schiff et al. first put forward the concept of chaos anticontrol [1], which involves discrete-time and continuoustime system for the chaos anticontrol. In the study of chaos anticontrol for continuous-time system, a lot of progress has been made and some methods of generating chaotic and hyperchaotic systems were found, such as the methods of time-delay feedback to chaos [2-4], topological conjugate to chaos [5], pulse control to chaos [6], parameter perturbation control to hyperchaos [7-9], and state feedback control to hyperchaos [10-13]. However, these methods of chaos anticontrol, which are based on the parameter "try," numerical simulation, and the Lyapunov index calculation, rely on the experience, lacking more theoretical basis. Therefore, it is still a challenge to construct a general approach of generating chaotic system.
Shil'nikov theorem is a beneficial tool to analyze the chaotic behavior of a nonlinear system [14], and it is also one of the most common decision theorems to determine whether chaos exists or not.

In 2004, Zhou et al. firstly constructed a threedimensional autonomous chaotic system which has only one equilibrium point [15] by using the homoclinic orbit Shil'nikov theorem. Then, $\mathrm{Li}$ and Chen proposed a method to construct a two-piecewise linear chaotic system by using the heteroclinic Shil'nikov theorem [16, 17]. Recently, Yu et al. also proposed a method for constructing multipiecewise linear chaotic systems $[18,19]$. However, the above studies lack further theoretical analysis and appropriate experimental verification.

In this paper, we use the heteroclinic Shil'nikov theorem to construct a kind of chaotic systems with both nonlinearities of two-piecewise linear and multipiecewise linear based 
on switching control method. Some basic dynamical characteristics of the constructed systems are analyzed theoretically and verified experimentally. Results of simulations and circuit experiments demonstrate the validity for the method of chaos anticontrol.

\section{Review of the Shil'nikov Theorem}

Consider the following three-dimensional autonomous dynamical system:

$$
\frac{d x}{d t}=f(x), \quad t \in R, x \in R^{3},
$$

where $f: R^{3} \rightarrow R^{3}$ belongs to $C^{r}(r \geq 2)$ and $x_{e} \in R^{3}$ is a hyperbolic saddle focus; that is, the eigenvalues of Jacobian $J=D f\left(x_{e}\right)$ are $\gamma$ and $\sigma \pm j \omega$, where $\gamma \sigma<0, \omega \neq 0, \gamma, \sigma$, and $\omega$ are all real constants.

Theorem 1 (the heteroclinic Shil'nikov theorem) (see [14]). Given the three-dimensional autonomous system shown in (1), let $x_{e 1}$ and $x_{e 2}$ be two distinct equilibrium points for (1). Suppose the following:

(1) Both $x_{e 1}$ and $x_{e 2}$ are saddle foci that satisfy the Shil'nikov inequality $\left|\gamma_{i}\right|>\left|\sigma_{i}\right|>0(i=1,2)$ with the further constraint $\gamma_{1} \gamma_{2}>0$ or $\sigma_{1} \sigma_{2}>0$.

(2) There is a heteroclinic loop, $H_{l}$, in which it joins $x_{e 1}$ to $x_{e 2}$ and is made up of two heteroclinic orbits $H_{i}(i=$ $1,2)$.

Both the original system (1) and its perturbed varieties exhibit the Smale horseshoe chaos.

In the following study, we will use the switching controller to construct piecewise linear chaotic systems based on this theorem.

\section{Two Fundamental Linear Systems}

For simplicity, we use the well-known Lorenz system to generate fundamental linear systems which are used to construct the piecewise linear chaotic systems.

The Lorenz system is described by

$$
\begin{aligned}
& \dot{x}=-a(x-y), \\
& \dot{y}=b x-x z-y, \\
& \dot{z}=-c z+x y,
\end{aligned}
$$

where $a=10, b=55$, and $c=5 / 3$. The equilibrium points of (2) are given as

$$
\begin{gathered}
O(0,0,0), \\
P^{+}(\sqrt{c(b-1)}, \sqrt{c(b-1)},(b-1)), \\
P^{-}(-\sqrt{c(b-1)},-\sqrt{c(b-1)},(b-1)) .
\end{gathered}
$$

From (3), we can see that the equilibrium points $P^{+}$ and $P^{-}$have certain symmetry. By linearizing (2) at the equilibrium points $P^{+}$and $P^{-}$, the linear equations are obtained, respectively, as follows:

$$
\begin{aligned}
& \left(\begin{array}{c}
\dot{x}_{1} \\
\dot{y}_{1} \\
\dot{z}_{1}
\end{array}\right) \\
& =\left(\begin{array}{ccc}
-a & a & 0 \\
1 & -1 & -\sqrt{c(b-1)} \\
\sqrt{c(b-1)} & \sqrt{c(b-1)} & -c
\end{array}\right)\left(\begin{array}{l}
x_{1} \\
y_{1} \\
z_{1}
\end{array}\right) \\
& =J_{1} X_{1} \text {, } \\
& \left(\begin{array}{c}
\dot{x}_{2} \\
\dot{y}_{2} \\
\dot{z}_{2}
\end{array}\right) \\
& =\left(\begin{array}{ccc}
-a & a & 0 \\
1 & -1 & \sqrt{c(b-1)} \\
-\sqrt{c(b-1)} & -\sqrt{c(b-1)} & -c
\end{array}\right)\left(\begin{array}{c}
x_{2} \\
y_{2} \\
z_{2}
\end{array}\right) \\
& =J_{2} X_{2} \text {. }
\end{aligned}
$$

Equations (4) and (5) are the required fundamental linear systems which are used to construct a piecewise linear chaotic system. The equilibrium points of the two linear systems are $\mathrm{O}_{1}(0,0,0)$ and $\mathrm{O}_{2}(0,0,0)$, and the corresponding eigenvalues are: $\gamma_{1}=\gamma_{2}=-14.0640$ and $\sigma_{1} \pm j \omega_{1}=\sigma_{2} \pm j \omega_{2}=$ $0.6987 \pm j 11.2915$. It can be seen that the equilibrium points $\mathrm{O}_{1}(0,0,0)$ and $\mathrm{O}_{2}(0,0,0)$ are all the saddle foci of index 2 and satisfy $\left|\gamma_{i}\right|>\left|\sigma_{i}\right|>0(i=1,2)$. According to the heteroclinic Shil'nikov theorem, there is chaos in the sense of Smale horseshoe as long as the equilibrium points of system (4) and system (5) are connected with the heteroclinic loop via a certain way.

The eigenvectors corresponding to the equilibrium points $\mathrm{O}_{1}(0,0,0)$ and $\mathrm{O}_{2}(0,0,0)$ of systems $(4)$ and (5) are, respectively,

$$
\begin{aligned}
& \mu_{1}=\left(\begin{array}{c}
0.8539 \\
-0.3470 \\
-0.3879
\end{array}\right), \\
& \eta_{1}=\left(\begin{array}{c}
-0.2421 \\
0.0617 \\
-0.7236
\end{array}\right) \pm j\left(\begin{array}{c}
0.2840 \\
0.5773 \\
0
\end{array}\right), \\
& \mu_{2}=\left(\begin{array}{c}
0.8539 \\
-0.3470 \\
0.3879
\end{array}\right), \\
& \eta_{2}=\left(\begin{array}{c}
-0.2421 \\
0.0617 \\
0.7236
\end{array}\right) \pm j\left(\begin{array}{c}
0.2840 \\
0.5773 \\
0
\end{array}\right) .
\end{aligned}
$$


At point $O_{1}(0,0,0)$, the mathematical expressions of the one-dimensional stable manifold $E^{S}\left(O_{1}\right)$ and the twodimensional unstable manifold $E^{U}\left(O_{1}\right)$ are given as

$$
\begin{gathered}
E^{S}\left(O_{1}\right): \quad \frac{x}{l_{1}}=\frac{y}{m_{1}}=\frac{z}{n_{1}}, \\
E^{U}\left(O_{1}\right): \quad A_{1} x+B_{1} y+C_{1} z=0,
\end{gathered}
$$

where the direction vectors of the stable manifold $E^{S}\left(O_{1}\right)$ are $l_{1}=0.8539, m_{1}=-0.3470$, and $n_{1}=-0.3879$ and the direction vectors of the unstable manifold $E^{U}\left(O_{1}\right)$ are given by $A_{1}=0.4177, B_{1}=-0.2055$, and $C_{1}=-0.1573$.

Similarly, the space straight line $E^{S}\left(\mathrm{O}_{2}\right)$ and the space plane $E^{U}\left(\mathrm{O}_{2}\right)$, which, respectively, correspond to the onedimensional stable manifolds and the two-dimensional unstable manifolds of equilibrium $\mathrm{O}_{2}(0,0,0)$ are described as

$$
\begin{gathered}
E^{S}\left(\mathrm{O}_{2}\right): \quad \frac{x}{l_{2}}=\frac{y}{m_{2}}=\frac{z}{n_{2}}, \\
E^{U}\left(\mathrm{O}_{2}\right): \quad A_{2} x+B_{2} y+C_{2} z=0,
\end{gathered}
$$

where the direction vectors of the stable manifold $E^{S}\left(\mathrm{O}_{2}\right)$ are $l_{2}=0.8539, m_{2}=-0.3470$, and $n_{2}=0.3879$ and those of unstable manifold $E^{U}\left(\mathrm{O}_{2}\right)$ are $A_{2}=-0.4177, B_{2}=0.2055$, and $C_{2}=-0.1573$.

\section{Construction of a Two-Piecewise Linear Chaotic System}

Next, we will study the existence conditions of the heteroclinic loop connecting the equilibrium points of system (4) and system (5), choosing the controller as $F(x, y, z)$ and the switching plane as $S=\{(x, y, z) \mid x=0\}$.

After translational transform for equilibrium points $O_{1}(0,0,0)$ and $O_{2}=(0,0,0)$, systems (4) and (5) can be changed into the following form:

$$
\begin{aligned}
& \left(\begin{array}{l}
\dot{x} \\
\dot{y} \\
\dot{z}
\end{array}\right) \\
& =\left(\begin{array}{ccc}
-a & a & 0 \\
1 & -1 & -\sqrt{c(b-1)} \\
\sqrt{c(b-1)} & \sqrt{c(b-1)} & -c
\end{array}\right)\left(\left(\begin{array}{l}
x \\
y \\
z
\end{array}\right)\right. \\
& -F(x, y, z))=J_{1}\left(\left(\begin{array}{l}
x \\
y \\
z
\end{array}\right)-F(x, y, z)\right), \\
& V \in V_{1}=\{(x, y, z) \mid x>0\},
\end{aligned}
$$

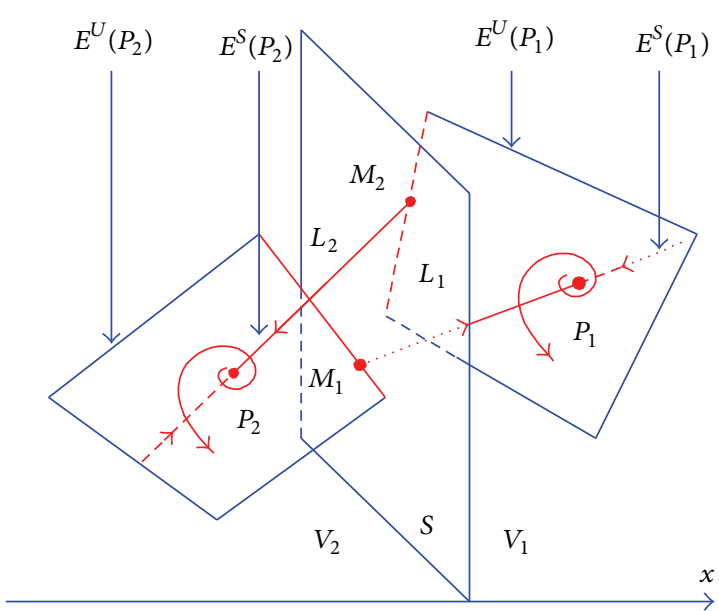

Figure 1: The eigenspaces corresponding to $P_{1}$ and $P_{2}$.

$$
\begin{aligned}
& \left(\begin{array}{l}
\dot{x} \\
\dot{y} \\
\dot{z}
\end{array}\right) \\
& =\left(\begin{array}{ccc}
-a & a & 0 \\
1 & -1 & \sqrt{c(b-1)} \\
-\sqrt{c(b-1)} & -\sqrt{c(b-1)} & -c
\end{array}\right)\left(\left(\begin{array}{l}
x \\
y \\
z
\end{array}\right)\right. \\
& -F(x, y, z))=J_{2}\left(\left(\begin{array}{c}
x \\
y \\
z
\end{array}\right)-F(x, y, z)\right), \\
& V \in V_{2}=\{(x, y, z) \mid x<0\},
\end{aligned}
$$

where $F(x, y, z)=\left[f_{1}(x, y, z), f_{2}(x, y, z), f_{3}(x, y, z)\right]^{T}$ is the switching controller whose concrete form is determined by the conditions of forming heteroclinic loops for system (9).

Let the equilibrium points of system (9) be $P_{1}\left(x_{1}, y_{1}, z_{1}\right) \in$ $V_{1}$ and $P_{2}\left(x_{2}, y_{2}, z_{2}\right) \in V_{2}$. At the equilibria $P_{1}$ and $P_{2}$, the space straight lines, $E^{\mathcal{S}}\left(P_{1}\right)$ and $E^{\mathcal{S}}\left(P_{2}\right)$, and the space plans, $E^{U}\left(P_{1}\right)$ and $E^{U}\left(P_{2}\right)$, are shown in Figure 1.

From (7), the space straight line equations of onedimensional stable manifold and space plan equation at equilibrium point $P_{1}\left(x_{1}, y_{1}, z_{1}\right) \in V_{1}$ are obtained as

$$
\begin{gathered}
E^{S}\left(P_{1}\right): \quad \frac{x-x_{1}}{l_{1}}=\frac{y-y_{1}}{m_{1}}=\frac{z-z_{1}}{n_{1}}, \\
E^{U}\left(P_{1}\right): \\
A_{1}\left(x-x_{1}\right)+B_{1}\left(y-y_{1}\right)+C_{1}\left(z-z_{1}\right)=0 .
\end{gathered}
$$


Similarly, from (8), we can get the space straight line equations of one-dimensional stable manifold and space plan equation at equilibrium point $P_{2}\left(x_{2}, y_{2}, z_{2}\right) \in V_{2}$ :

$$
\begin{gathered}
E^{S}\left(P_{2}\right): \quad \frac{x-x_{2}}{l_{2}}=\frac{y-y_{2}}{m_{2}}=\frac{z-z_{2}}{n_{2}}, \\
E^{U}\left(P_{2}\right): \\
A_{2}\left(x-x_{2}\right)+B_{2}\left(y-y_{2}\right)+C_{2}\left(z-z_{2}\right)=0 .
\end{gathered}
$$

Let $M_{1}$ and $M_{2}\left(L_{1}\right.$ and $\left.L_{2}\right)$ be the intersection points (lines) of $E^{S}\left(P_{1}\right), E^{S}\left(P_{2}\right),\left(E^{U}\left(P_{1}\right)\right.$ and $\left.E^{U}\left(P_{2}\right)\right)$ with the switching plane $S=\{(x, y, z) \mid x=0\}$; that is,

$$
\begin{aligned}
& M_{1}=E^{S}\left(P_{1}\right) \cap S:\left(0, y_{1}-\frac{m_{1}}{l_{1}} x_{1}, z_{1}-\frac{n_{1}}{l_{1}} x_{1}\right) \text {, } \\
& M_{2}=E^{S}\left(P_{2}\right) \cap S:\left(0, y_{2}-\frac{m_{2}}{l_{2}} x_{2}, z_{2}-\frac{n_{2}}{l_{2}} x_{2}\right) \text {, } \\
& L_{1}=E^{U}\left(P_{1}\right) \cap S: \\
& \left\{\begin{array}{l}
A_{1}\left(x-x_{1}\right)+B_{1}\left(y-y_{1}\right)+C_{1}\left(z-z_{1}\right)=0 \\
x=0
\end{array}\right. \\
& L_{2}=E^{U}\left(P_{2}\right) \cap S: \\
& \left\{\begin{array}{l}
A_{2}\left(x-x_{2}\right)+B_{2}\left(y-y_{2}\right)+C_{2}\left(z-z_{2}\right)=0 \\
x=0 .
\end{array}\right.
\end{aligned}
$$

If $M_{1}$ lies on $L_{2}$, there exists a heteroclinic orbit $H_{1}=$ $E^{U}\left(P_{2}\right) \cup M_{1} \cup E^{S}\left(P_{1}\right)$ from $P_{2}$ to $P_{1}$. Similarly, if $M_{2}$ lies on $L_{1}$, then there exists a heteroclinic orbit $H_{2}=E^{U}\left(P_{1}\right) \cup M_{2} \cup$ $E^{S}\left(P_{2}\right)$ from $P_{1}$ to $P_{2}$. If the above two conditions are satisfied simultaneously, there must exist a heteroclinic loop, which consists of heteroclinic orbits $H_{1}$ and $H_{2}$ and connects $P_{1}$ with $P_{2}$. According to the heteroclinic Shil'nikov theorem, system (9) exhibits the Smale horseshoe chaos.

In the following parts, the conditions that the coordinates of the equilibrium point must meet will be analyzed when the heteroclinic loop of system (9) exists. From the invariance of transformation $(x, y, z) \rightarrow(-x,-y, z)$, one can assume that the coordinates of the equilibria $P_{1}=\left(x_{1}, y_{1}, z_{1}\right)$ and $P_{2}\left(x_{2}, y_{2}, z_{2}\right)$ of system (9) satisfy the following formula:

$$
\begin{aligned}
& x_{1}=-x_{2}=x_{0}, \\
& y_{1}=-y_{2}=y_{0}, \\
& z_{1}=z_{2}=z_{0} .
\end{aligned}
$$

From (12) and (13), one can get the necessary conditions of $M_{1} \in L_{2}$ and $M_{2} \in L_{1}$ as

$$
\frac{A_{2} l_{1}-B_{2} m_{1}-C_{2} n_{1}}{B_{2} l_{1}}=\frac{A_{1} l_{2}-B_{1} m_{2}-C_{1} n_{2}}{B_{1} l_{2}} .
$$

When the parameters $A_{i}, B_{i}, C_{i}, l_{i}, m_{i}$, and $n_{i}(i=1,2)$ satisfy (14), from (12) and (13), $y_{0}$ can be obtained:

$$
\begin{aligned}
y_{0} & =\frac{\left(A_{2} l_{1}-B_{2} m_{1}-C_{2} n_{1}\right) x_{0}}{2 B_{2} l_{1}} \\
& =-\frac{\left(A_{1} l_{2}-B_{1} m_{2}-C_{1} n_{2}\right) x_{0}}{2 B_{1} l_{2}} .
\end{aligned}
$$

Equation (15) indicates that $y_{0}$ depends on the value of $x_{0}$ but is not associated with $z_{0}$ that can take any value. Here, let $x_{1}=-x_{2}=x_{0}=1$ and $z_{1}=z_{2}=z_{0}=0$; we can get $y_{0}=0.9870$. And when a heteroclinic loop of system (9) exists, the coordinates of the equilibria $P_{1}=\left(x_{1}, y_{1}, z_{1}\right)$ and $P_{2}=\left(x_{2}, y_{2}, z_{2}\right)$ of system (9) are

$$
\begin{aligned}
& P_{1}=\left(x_{1}, y_{1}, z_{1}\right)=\left(x_{0}, y_{0}, z_{0}\right)=(1,0.9870,0), \\
& P_{2}=\left(x_{2}, y_{2}, z_{2}\right)=\left(-x_{0},-y_{0}, z_{0}\right)=(-1,-0.9870,0) .
\end{aligned}
$$

To make the coordinates of the equilibria of system (9) satisfy (16), the controller of system (9) should be selected as following form:

$$
F(x, y, z)=\left(\begin{array}{l}
f_{1}(x, y, z) \\
f_{2}(x, y, z) \\
f_{3}(x, y, z)
\end{array}\right)=\left(\begin{array}{l}
x_{0} \operatorname{sgn}(x) \\
y_{0} \operatorname{sgn}(x) \\
z_{0} \operatorname{sgn}(z)
\end{array}\right)
$$

where $x_{0}=1, y_{0}=0.9870$, and $z_{0}=0$.

According to the above analysis and by introducing switching function $T=T(x, y, z)=\operatorname{sgn}(x)$ to (9), it is expressed that

$$
\begin{aligned}
& \left(\begin{array}{l}
\dot{x} \\
\dot{y} \\
\dot{z}
\end{array}\right) \\
& =\left(\begin{array}{ccc}
-a & a & 0 \\
1 & -1 & -T \sqrt{c(b-1)} \\
T \sqrt{c(b-1)} & T \sqrt{c(b-1)} & -c
\end{array}\right)\left(\left(\begin{array}{l}
x \\
y \\
z
\end{array}\right)\right. \\
& -F(x, y, z)) .
\end{aligned}
$$

Equation (18) is the expected two-piecewise linear chaotic system, which has a double-wing chaotic attractor, shown in Figure 2.

Based on (15), we can construct a multipiecewise linear chaotic system via alternate translational transform of the two fundamental linear systems and heteroclinic loop construction of adjacent equilibria for the multipiecewise linear system. 


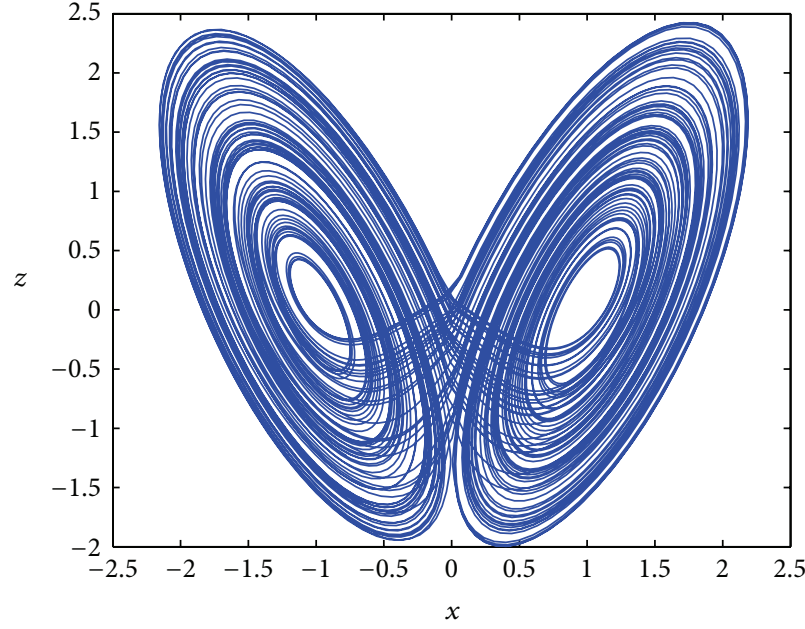

(a)

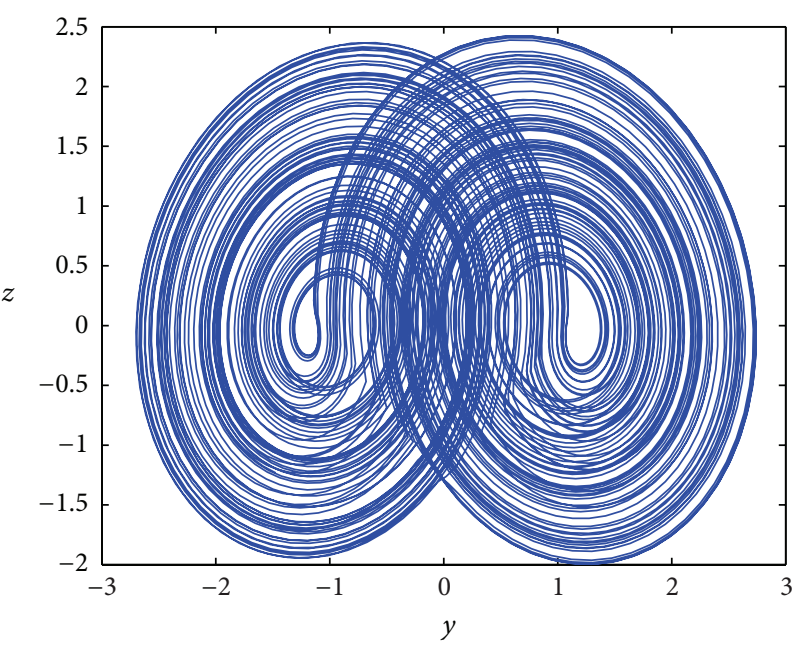

(b)

FIgURE 2: Two-wing chaotic attractor of system (18). (a) $x-z$ plane projection. (b) $y$ - $z$ plane projection.

The mathematical expression for the multilinear chaotic systems is described as

$$
\begin{aligned}
& \left(\begin{array}{c}
\dot{x} \\
\dot{y} \\
\dot{z}
\end{array}\right) \\
& =\left(\begin{array}{ccc}
-a & a & 0 \\
1 & -1 & -T \sqrt{c(b-1)} \\
T \sqrt{c(b-1)} & T \sqrt{c(b-1)} & -c
\end{array}\right)\left(\left(\begin{array}{l}
x \\
y \\
z
\end{array}\right)\right. \\
& -F(x, y, z)),
\end{aligned}
$$

where

$$
\begin{aligned}
& F(x, y, z)=\left(\begin{array}{l}
f_{1}(x, y, z) \\
f_{2}(x, y, z) \\
f_{3}(x, y, z)
\end{array}\right) \\
& =\left(\begin{array}{c}
x_{0} \operatorname{sgn}(x)+\sum_{m=1}^{M} x_{0}\left[\operatorname{sgn}\left(x+2 m x_{0}\right)+\operatorname{sgn}\left(x-2 m x_{0}\right)\right] \\
y_{0} \operatorname{sgn}(x)+\sum_{m=1}^{M} y_{0}\left[\operatorname{sgn}\left(x+2 m x_{0}\right)+\operatorname{sgn}\left(x-2 m x_{0}\right)\right] \\
z_{0} \operatorname{sgn}(z)+\sum_{n=1}^{N} z_{0}\left[\operatorname{sgn}\left(z+2 n z_{0}\right)+\operatorname{sgn}\left(z-2 n z_{0}\right)\right]
\end{array}\right), \\
& T=T(x, y, z) \\
& =\operatorname{sgn}(x)+\sum_{m=1}^{M}-1^{m}\left[\operatorname{sgn}\left(x+2 m x_{0}\right)+\operatorname{sgn}\left(x-2 m x_{0}\right)\right] .
\end{aligned}
$$

When $z_{0}=0$ and $f_{3}(x, y, z)=0$, system (19) can generate a chaotic attractor with $(2 M+2)$-wings, while when $z_{0} \neq 0$, a chaotic attractor with $(2 M+2) \times(2 N+2)$-grid wings can be obtained. Figure 3 (a) shows a 6-wing chaotic attractor with $x_{0}=1, y_{0}=0.9870, z_{0}=0$, and $M=2$. When $x_{0}=1$, $y_{0}=0.9870, z_{0}=1.6, M=2$, and $N=0$, a $6 \times 2$-grid wing chaotic attractor is found, which is shown in Figure 3(b).

\section{Basic Dynamics of Two-Piecewise Linear Chaotic System}

In this section, some basic dynamics of system (18) will be analyzed, including the dissipation, the Lyapunov exponents, and the bifurcation diagram.

5.1. Dissipation of System. It is well known that a chaotic system is a dissipative system. According to (17) and (18), we can get the dissipation character of the two-piecewise linear system from the following equation:

$$
\nabla V=\frac{\partial \dot{x}}{\partial x}+\frac{\partial \dot{y}}{\partial y}+\frac{\partial \dot{z}}{\partial z}=-a-1-c=-\frac{38}{3}<0 .
$$

5.2. Lyapunov Exponent Spectrum and Bifurcation Diagram. Lyapunov exponent is the most direct evidence to determine a chaotic system. Figure 4 shows the Lyapunov exponent spectrum versus parameter $a$ in system (18), from which we can see one of the Lyapunov exponents is always positive when the parameter $a$ changes in a large range, indicating that system (18) is chaotic. Larger range of chaotic parameter can provide larger space of key which can increase the difficulty of unmasking signals and improve the security of communications. Therefore, system (18) has significance if it is applied to secure communications as pseudorandom signal source.

Figure 5 shows the bifurcation diagram versus parameter $a$ of system (18), from which we can see that system (18) is chaotic apart from a few tiny periodic windows. From Figure 5, we also can see the road of system (18) to chaos. System (18) evolves into chaotic state not through perioddoubling bifurcation but directly from stable state or from periodic motion, while the system parameters vary. 


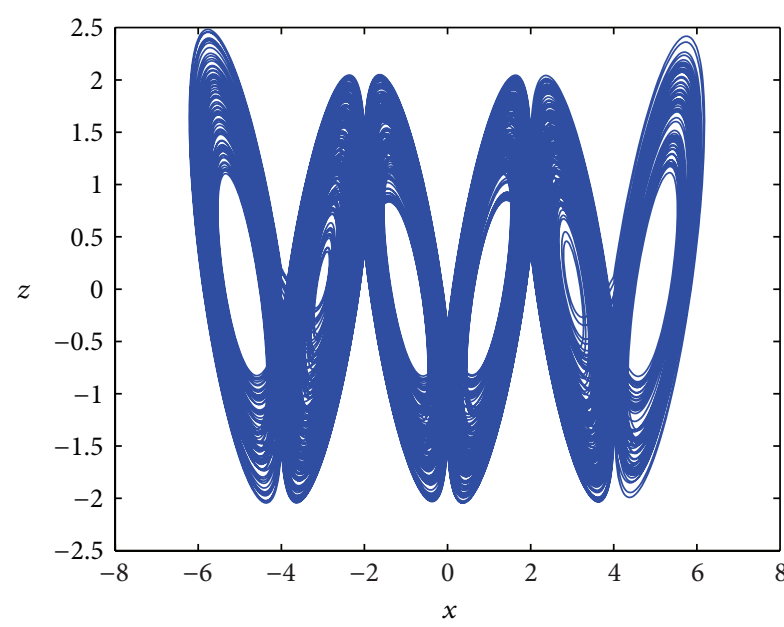

(a)

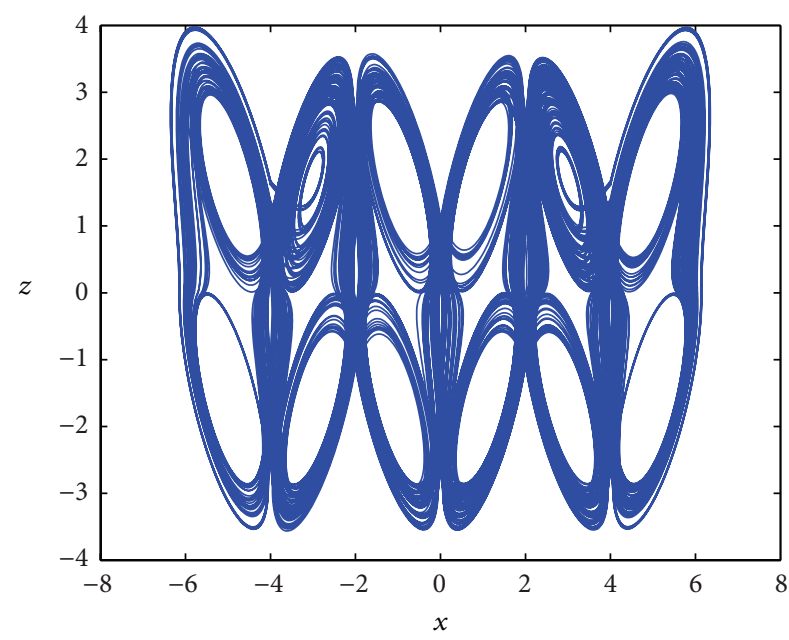

(b)

FIGURE 3: The $x-z$ plane projections of multiwing and grid wing chaotic attractors of system (19). (a) Six-wing chaotic attractor. (b) $6 \times 2$-grid wing chaotic attractor.

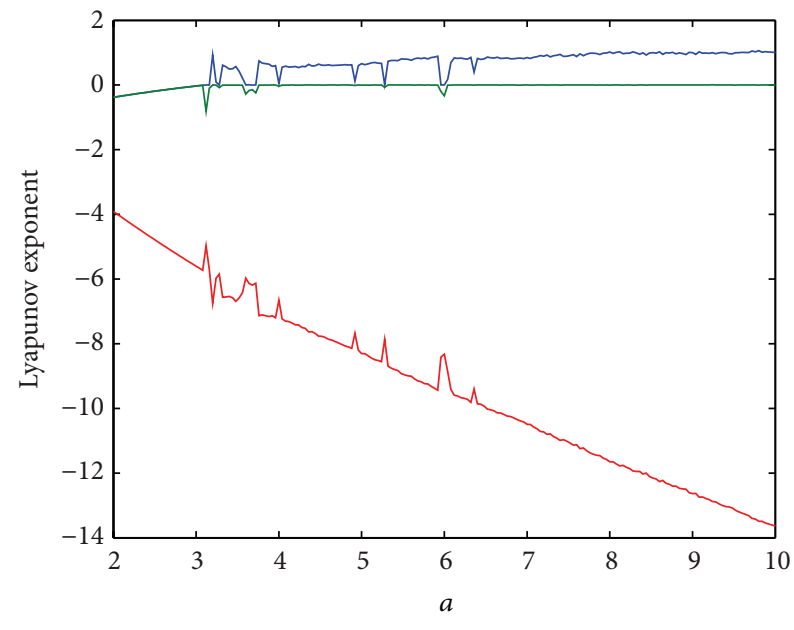

FIGURE 4: The diagram of LE versus parameter $a$ of system (18).

\section{Circuit Implementation of the Multipiecewise Linear Chaotic System}

According to (19)-(20), a circuit of realizing system (19) is shown in Figure 6, which can generate 6-wing chaotic attractor and $6 \times 2$-grid wing chaotic attractor. The modular circuits for implementing functions $\pm\left(x-f_{1}\right), \pm\left(y-f_{2}\right)$, $\left(z-f_{3}\right)$, and $T$ are designed and shown in Figure 7 , which consist of adders, inverters, multipliers, and integrators. In the modular circuits, the model of operational amplifiers is TL082 and that of multipliers is AD633.

According to Figure 6, the circuit equation of system (19) can be obtained:

$$
\frac{d x}{d t}=\frac{1}{R C}\left[-\frac{R}{R_{1}}\left(x-f_{1}\right)+\frac{R}{R_{2}}\left(y-f_{2}\right)\right],
$$

$$
\begin{aligned}
\frac{d y}{d t} & =\frac{1}{R C}\left[\frac{R}{R_{3}}\left(x-f_{1}\right)-\frac{R}{R_{4}}\left(y-f_{2}\right)\right. \\
& \left.-T \frac{R}{10 R_{5}}\left(z-f_{3}\right)\right], \\
\frac{d z}{d t} & =\frac{1}{R C}\left[T \frac{R}{10 R_{6}}\left(x-f_{1}\right)+T \frac{R}{10 R_{7}}\left(y-f_{2}\right)\right. \\
- & \left.\frac{R}{R_{8}}\left(z-f_{3}\right)\right] .
\end{aligned}
$$

Let $t=10 R C t^{\prime}$; one has

$$
\begin{aligned}
& \frac{d x}{10 R C d t^{\prime}}=\frac{1}{R C}\left[-\frac{R}{R_{1}}\left(x-f_{1}\right)+\frac{R}{R_{2}}\left(y-f_{2}\right)\right], \\
& \frac{d y}{10 R C d t^{\prime}}=\frac{1}{R C}\left[\frac{R}{R_{3}}\left(x-f_{1}\right)-\frac{R}{R_{4}}\left(y-f_{2}\right)\right. \\
& \left.-T \frac{R}{10 R_{5}}\left(z-f_{3}\right)\right], \\
& \frac{d z}{10 R C d t^{\prime}}=\frac{1}{R C}\left[T \frac{R}{10 R_{6}}\left(x-f_{1}\right)+T \frac{R}{10 R_{7}}\left(y-f_{2}\right)\right. \\
& \left.-\frac{R}{R_{8}}\left(z-f_{3}\right)\right] .
\end{aligned}
$$

Let again $t^{\prime}=t$; (23) can be changed as follows:

$$
\begin{aligned}
\frac{d x}{d t} & =10\left[-\frac{R}{R_{1}}\left(x-f_{1}\right)+\frac{R}{R_{2}}\left(y-f_{2}\right)\right], \\
\frac{d y}{d t} & =10\left[\frac{R}{R_{3}}\left(x-f_{1}\right)-\frac{R}{R_{4}}\left(y-f_{2}\right)\right. \\
- & \left.T \frac{R}{10 R_{5}}\left(z-f_{3}\right)\right],
\end{aligned}
$$




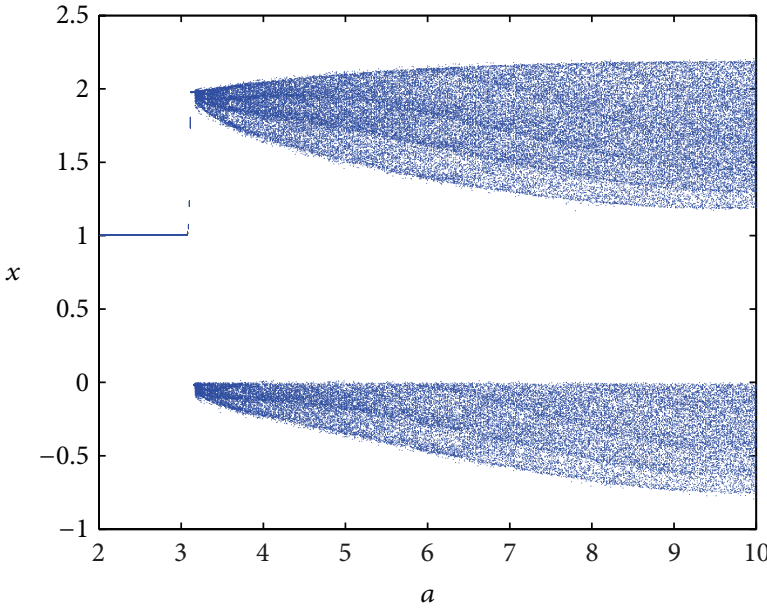

(a)

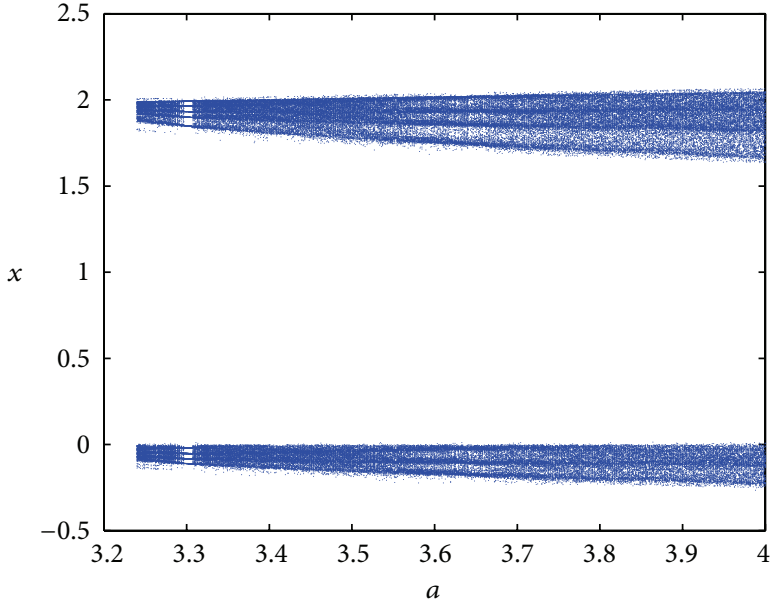

(b)

Figure 5: The bifurcation diagram versus parameter $a$ of system (18). (a) $a$ varies from 2 to 10. (b) $a$ varies from 3.24 to 4 .
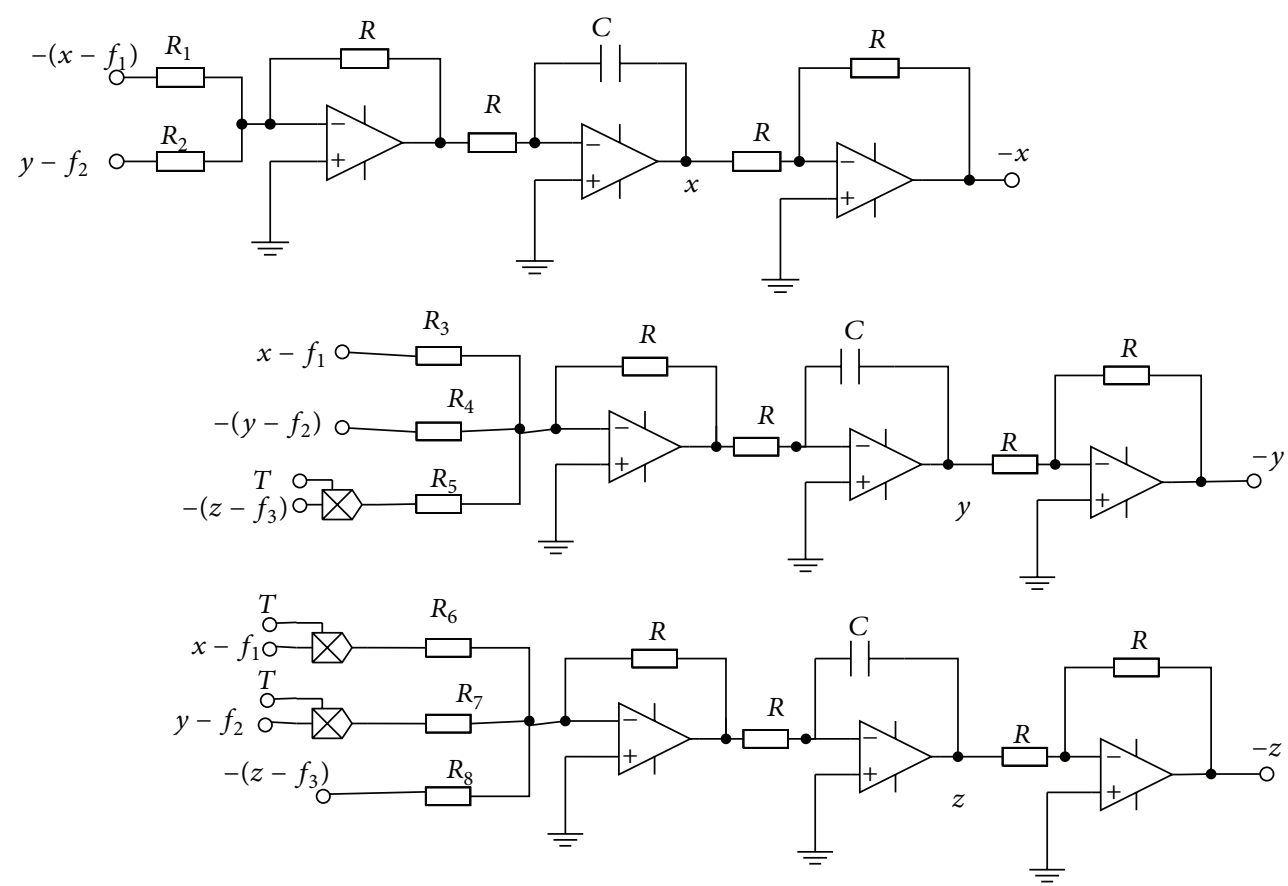

FIgURE 6: Module-based circuit diagram for realizing system (19).

$$
\begin{aligned}
\frac{d z}{d t} & =10\left[T \frac{R}{10 R_{6}}\left(x-f_{1}\right)+T \frac{R}{10 R_{7}}\left(y-f_{2}\right)\right. \\
& \left.-\frac{R}{R_{8}}\left(z-f_{3}\right)\right] .
\end{aligned}
$$

Comparing (24) with (19), one has

$$
\begin{aligned}
& \frac{10 R}{R_{1}}=\frac{10 R}{R_{2}}=a, \\
& \frac{10 R}{R_{3}}=\frac{10 R}{R_{4}}=1,
\end{aligned}
$$

$$
\begin{aligned}
\frac{R}{R_{5}} & =\frac{R}{R_{6}}=\frac{R}{R_{7}}=\sqrt{c(b-1)}, \\
\frac{10 R}{R_{8}} & =c .
\end{aligned}
$$

When $a=10, b=55, c=5 / 3$, and $R=10 \mathrm{k} \Omega$, the resistance values in Figure 6 can be obtained:

$$
\begin{aligned}
& R_{1}=R_{2}=10 \mathrm{k} \Omega, \\
& R_{3}=R_{4}=100 \mathrm{k} \Omega, \\
& R_{5}=R_{6}=R_{7}=1.054 \mathrm{k} \Omega, \\
& R_{8}=60 \mathrm{k} \Omega .
\end{aligned}
$$



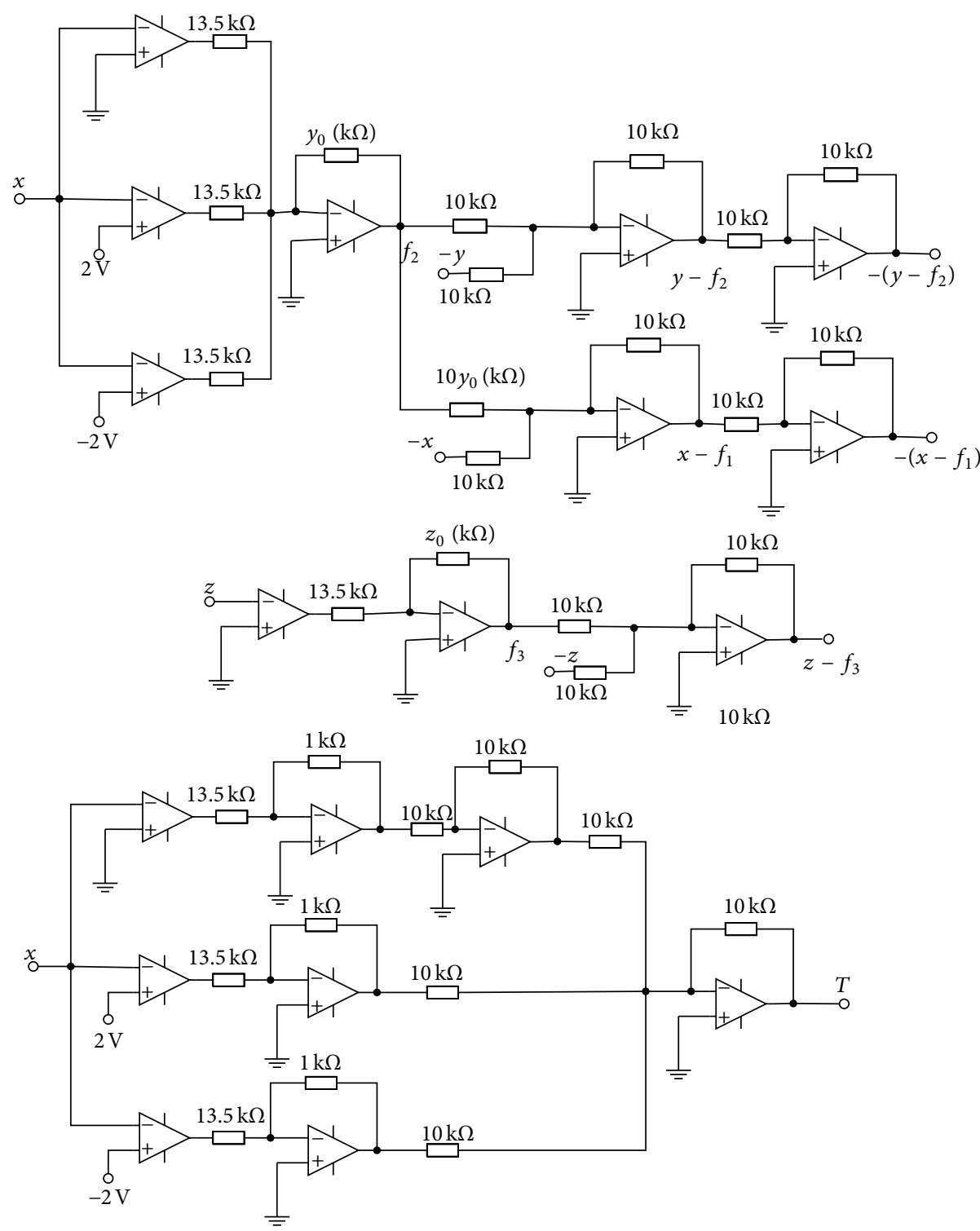

FIgURE 7: Module-based circuit diagram for realizing functions $\pm\left(x-f_{1}\right), \pm\left(y-f_{2}\right), \pm\left(z-f_{3}\right)$, and $T$.

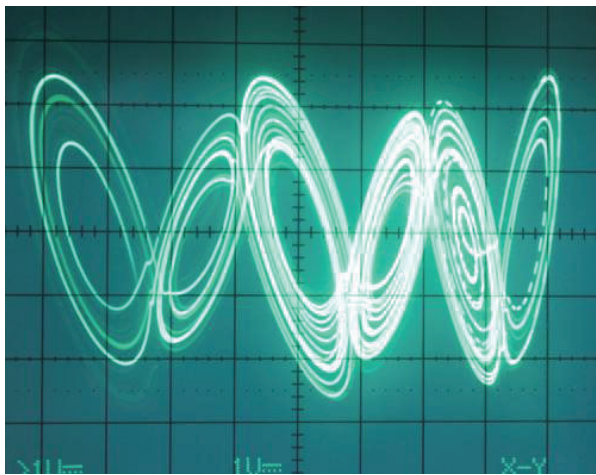

(a)

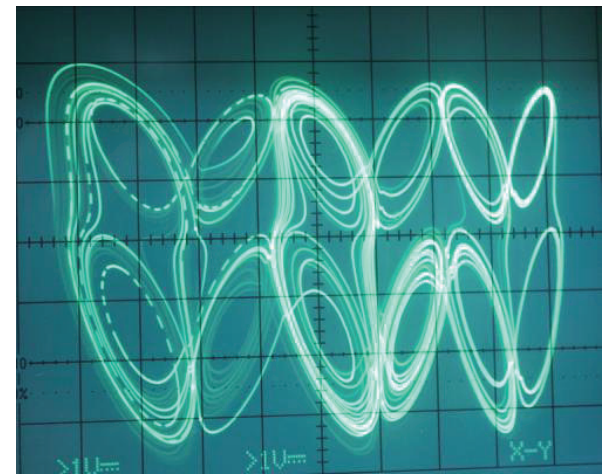

(b)

FIGURE 8: Experimental observation of multiwing and grid wing chaotic attractors of system (19). (a) Six-wing chaotic attractor. (b) $6 \times 2$-grid wing chaotic attractor. 
When the capacitance $C=10 \mathrm{nF}$, the 6 -wing chaotic attractors and $6 \times 2$-grid wing chaotic attractors obtained observed from oscilloscope are shown in Figure 8.

\section{Conclusion}

Based on the heteroclinic Shil'nikov theorem, a method for constructing a kind of multipiecewise linear chaotic system has been proposed via switching control in this paper. By using this method, a two-piecewise linear and a multipiecewise linear chaotic systems are constructed.

It is worth pointing that this method of constructing chaotic system has general significance. It does not only suit Lorenz system in this paper but also can be applied to the three-dimensional autonomous system which has saddle focus equilibrium points $P_{1,2}( \pm x, \pm y, z)$ and satisfies $\gamma_{1} \gamma_{2}>0$ or $\sigma_{1} \sigma_{2}>0$ and $\left|\gamma_{i}\right|>\left|\sigma_{i}\right|>0(i=1,2)$. In addition, because the circuit structure of implementing the multipiecewise linear chaotic system is simple and easy to implement, this chaos anticontrol method has more potential applications than other chaotic anticontrol methods of generating multiwings and multigrid wing chaotic attractors in engineering applications.

\section{Conflict of Interests}

The authors declare that there is no conflict of interests regarding the publication of this paper.

\section{Acknowledgments}

This work was supported by the National Natural Science Foundation of China (Grant nos. 61271064 and 60971046), the Natural Science Foundation of Zhejiang Province, China (Grant no. LZ12F01001), the Research Foundation of Binzhou University (Grant no. BZXYG1501), and the Program for Zhejiang Leading Team of S \& T Innovation, China (Grant no. 2010R50010-07).

\section{References}

[1] S. J. Schiff, K. Jerger, D. H. Duong et al., "Controlling chaos in the brain," Nature, vol. 363, pp. 411-417, 1994.

[2] X. F. Wang, G. Chen, and X. Yu, "Anticontrol of chaos in continuous-time systems via time-delay feedback," Chaos, vol. 10, no. 4, pp. 771-779, 2000.

[3] X. F. Wang, G. Chen, and K. F. Man, "Making a continuous-time minimum-phase system chaotic by using time-delay feedback," IEEE Transactions on Circuits and Systems I: Fundamental Theory and Applications, vol. 48, no. 5, pp. 641-645, 2001.

[4] T. S. Zhou, G. R. Chen, and Q. G. Yang, "A simple time-delay feedback anticontrol method made rigorous," Chaos, vol. 14, no. 3, pp. 662-668, 2004.

[5] X. F. Wang and G. R. Chen, "Generating topologically conjugate chaotic systems via feedback control," IEEE Transactions on Circuits and Systems I, vol. 50, no. 6, pp. 812-817, 2003.

[6] L. Yang, Z. R. Liu, and G. R. Chen, "Chaotifying a continuoustime system via impulsive input," International Journal of
Bifurcation and Chaos in Applied Sciences and Engineering, vol. 12, no. 5, pp. 1121-1128, 2002.

[7] Y. X. Li, G. R. Chen, and W. K. S. Tang, "Controlling a unified chaotic system to hyperchaotic," IEEE Transactions on Circuits and Systems II: Express Briefs, vol. 52, no. 4, pp. 204-207, 2005.

[8] K. H. Sun, X. Liu, C. X. Zhu, and J. C. Sprott, "Hyperchaos and hyperchaos control of the sinusoidally forced simplified Lorenz system," Nonlinear Dynamics, vol. 69, no. 3, pp. 1383-1391, 2012.

[9] C. Shi-Jian, C. Zeng-Qiang, and W. Wen-Juan, "Circuit implementation and multiform intermittency in a hyper-chaotic model extended from the Lorenz system," Chinese Physics B, vol. 18, no. 5, pp. 1792-1800, 2009.

[10] Y. X. Li, W. K. S. Tang, and G. R. Chen, "Hyperchaos evolved from the generalized Lorenz equation," International Journal of Circuit Theory and Applications, vol. 33, no. 4, pp. 235-251, 2005.

[11] A. M. Chen, J. A. Lu, J. H. Lü, and S. M. Yu, "Generating hyperchaotic Lü attractor via state feedback control," Physica A: Statistical Mechanics and Its Applications, vol. 364, pp. 103-110, 2006.

[12] K. H. Sun, X. Liu, and C. X. Zhu, "Dynamics of a strengthened chaotic system and its circuit implementation," Chinese Journal of Electronics, vol. 23, no. 2, pp. 353-356, 2014.

[13] H. L. Xi, S. M. Yu, C. Zhang, and Y. Sun, "Generation and implementation of hyperchaotic chua system via state feedback control," International Journal of Bifurcation and Chaos, vol. 22, no. 5, pp. 56-64, 2012.

[14] C. P. Silva, “Shil'nikov's theorem-a tutorial," IEEE Transactions on Circuits and Systems I: Fundamental Theory and Applications, vol. 40, no. 10, pp. 675-682, 1993.

[15] T. S. Zhou, G. R. Chen, and Q. G. Yang, "Constructing a new chaotic system based on the Silnikov criterion," Chaos, Solitons and Fractals, vol. 19, no. 4, pp. 985-993, 2004.

[16] G. L. Li and X. Y. Chen, "Constructing piecewise linear chaotic system based on the heteroclinic Shil'nikov theorem," Communications in Nonlinear Science and Numerical Simulation, vol. 14, no. 1, pp. 194-203, 2009.

[17] G.-L. Li and X.-Y. Chen, "Design of piecewise linear chaotic system," Acta Electronica Sinica, vol. 36, no. 9, pp. 1814-1818, 2008.

[18] C.-X. Zhang, S.-M. Yu, and Y. Zhang, "Design and realization of multi-wing chaotic attractors via switching control," International Journal of Modern Physics B, vol. 25, no. 16, pp. 2183-2194, 2011.

[19] S. M. Yu, J. H. Lü, G. R. Chen, and X. Yu, "Generating grid multiwing chaotic attractors by constructing heteroclinic loops into switching systems," IEEE Transactions on Circuits and Systems II: Express Briefs, vol. 58, no. 5, pp. 314-318, 2011. 

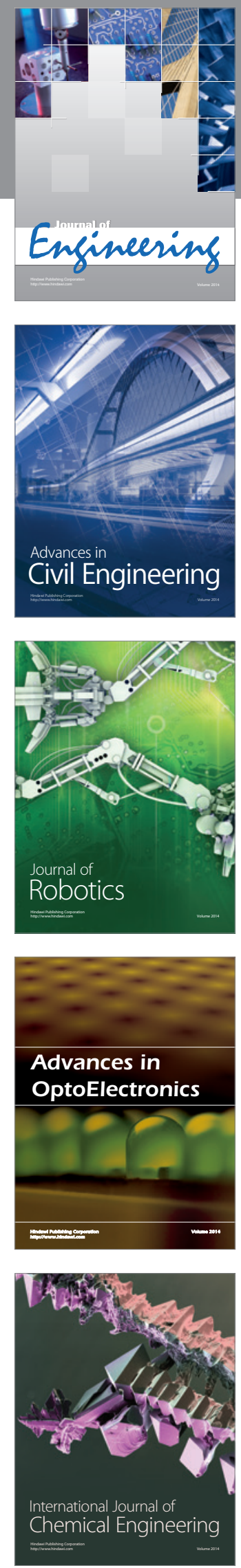

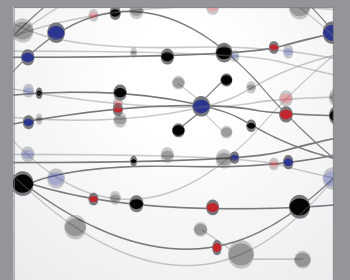

The Scientific World Journal
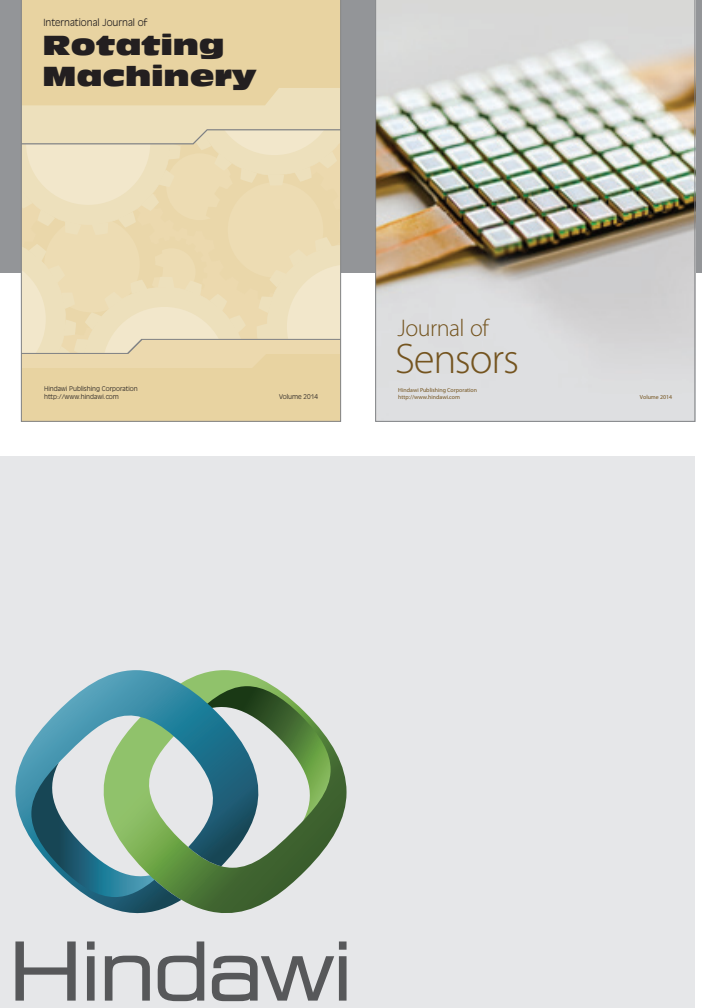

Submit your manuscripts at http://www.hindawi.com
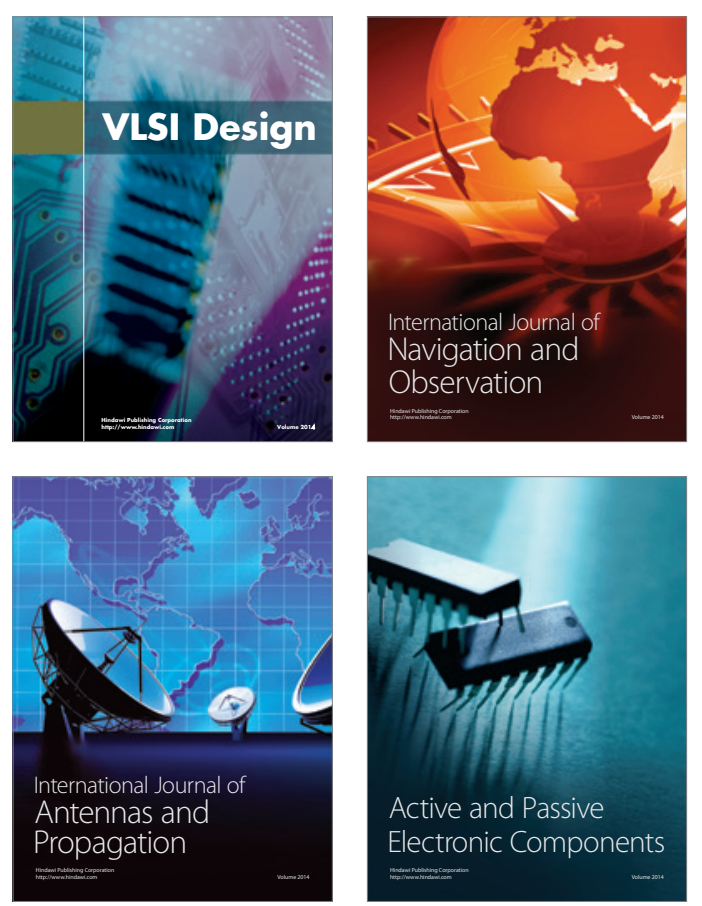
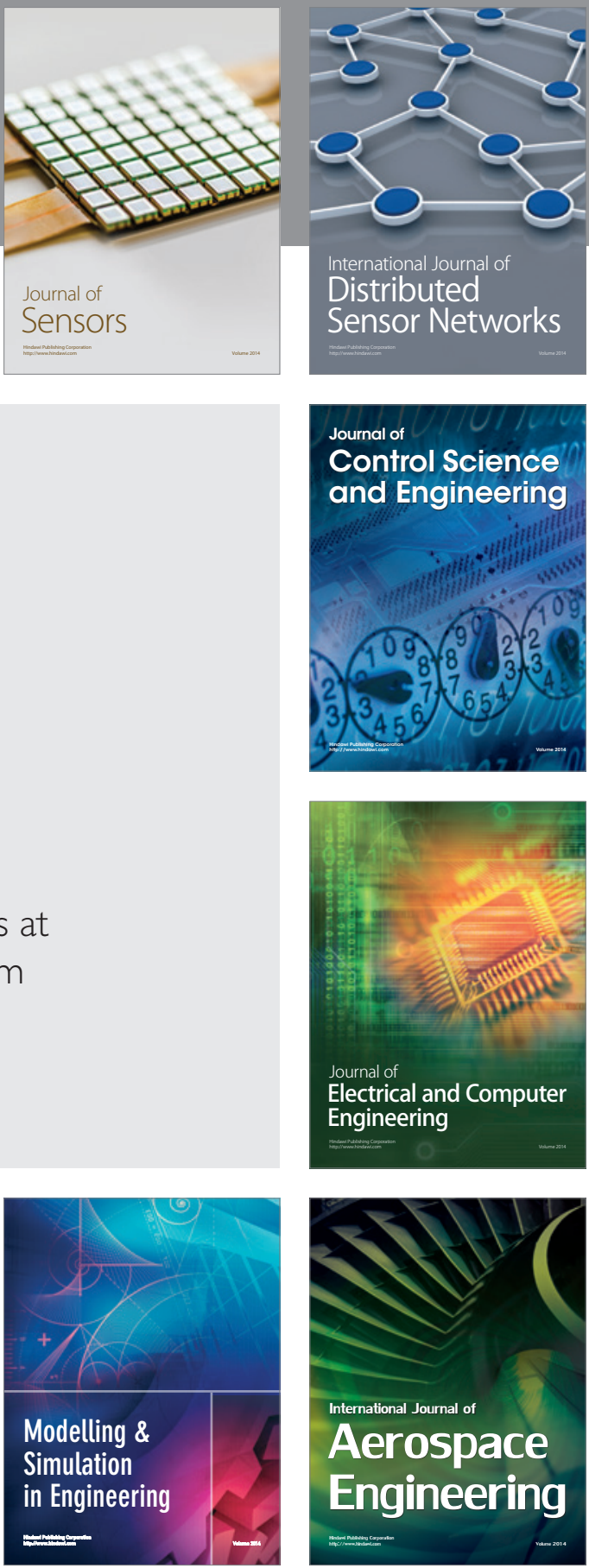

Journal of

Control Science

and Engineering
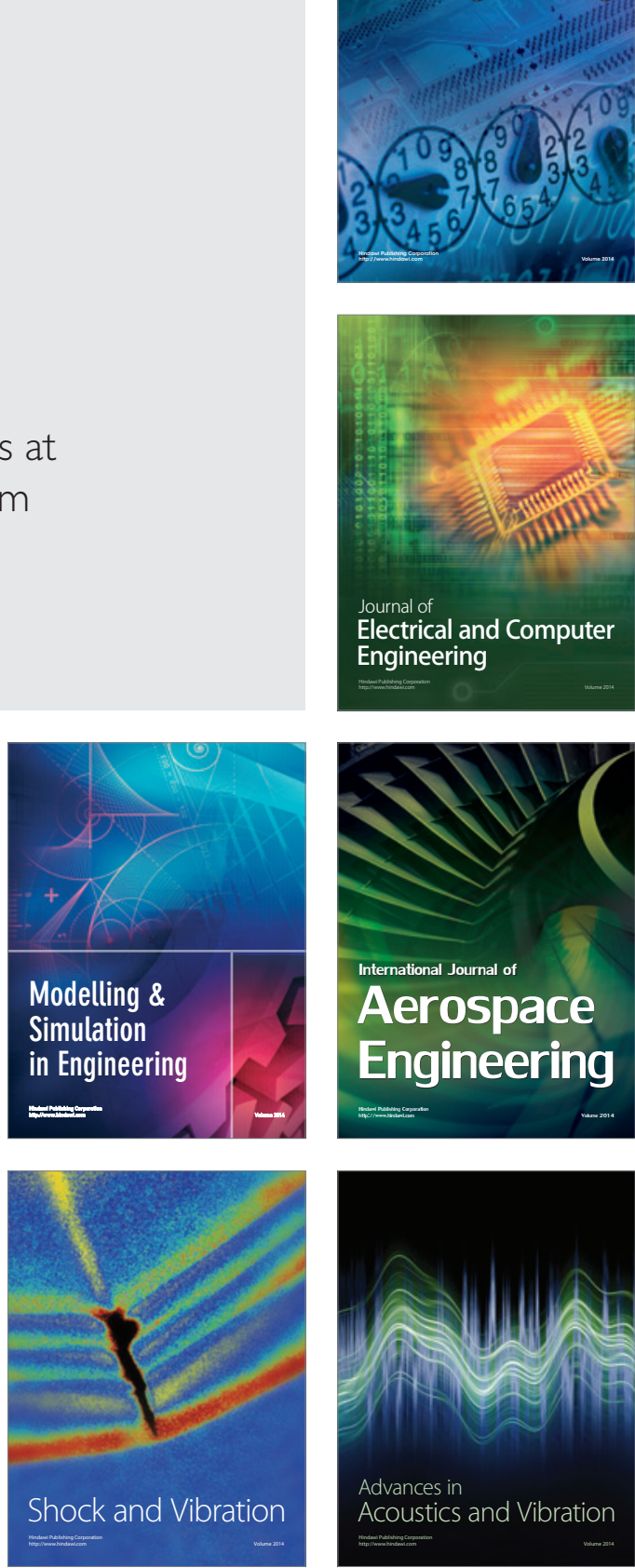